\title{
La liaison Main-Damube favorise la navigation et l'économie de l'eau
}

\section{How the Main-Danube link facilitates navigation and saves water}

\author{
Burkart Ruimelin
}

Membre du Comité de Direction de la Société Rhin-Main-Danube, Munich

\section{La voie navigable Main-Danube}

Par la liaison entre la vallée du Rhin et l'espace danubien à travers le Jura franconien se réalisera un vieux rêve européen. Les travaux de la Société Rhin-MainDanube (RMD) battent leur plein. La demière phase dans la réalisation de cet ouvrage de communication approche.

\section{Principes d'étude}

Il se conçoit que le tracé de cette voie de navigation suive dans toute la mesure du possible les vallées de cours d'eau, à moins que des agglomérations, ponts de chemin de fer ou similaires ne s'y opposent. Comme solution optimale fut retenu, après de nombreuses études et modifications, dont la dernière en novembre 1976 , le tracé ci-après entre Bamberg et Kelheim. Entre Bamberg et Forchheim, ce tracé suit, sur une longueur de $31,9 \mathrm{~km}$, la vallée de la Regnitz dont il emprunte en partie le lit, pour s'élever de $23,7 \mathrm{~m}$. C'est là que commence la section en canal, longue de $105 \mathrm{~km}$ avec une dénivellation de $58 \mathrm{~m}$ jusqu'au port de Nuremberg puis de $93,5 \mathrm{~m}$ jusqu'au bief de partage pour descendre ensuite de $51 \mathrm{~m}$, à travers la vallée de la Sulz et la vallée d'Ottmaring, jusqu'à l'Altmühl, près de Dietfurt. Puis le tracé emprunte la vallée de l'Altmühl et atteint après un parcours de $34,1 \mathrm{~km}$ et une dénivellation de $16,8 \mathrm{~m}$, le Danube près de Kelheim.

La variante envisagée dans les années 50 et prévoyant 3 ascenseurs à bateaux à Rednitzhembach, Hilpoltstein et Dietfurt fut abandonnée en juillet 1969 à la suite d'importantes études sur la qualité du sous-sol, la sécurité de fonctionnement, la capacité, le coût de construction et les frais d'exploitation et de maintenance de telles intallations. En outre, la longueur utile de ces ascenseurs est limitée à environ $100 \mathrm{~m}$. D'autre part, d'après les expériences recueillies sur les écluses d'Erlangen et de Kriegenbrunn et les essais sur modèles exécutés par le Professeur Dr. Mosonyi à Karlsruhe, la réalisation d'écluses à grande chute avec bassins d'épargne latéraux et une hauteur de chute d'environ $25 \mathrm{~m}$ ne paraissait plus poser de problèmes. La décision en faveur d'écluses de $12 \mathrm{~m}$ de largeur et de $190 \mathrm{~m}$ de longueur utile fut en outre influencée par le fait que des ascenseurs constituent un élément étranger dans des échelles d'écluses et exigent une maintenance coûteuse.

Les études ont fait ressortir que la section mouillée aussi bien du canal que de la Regnitz et de l'Altmühl doit, si possible, être égale à 7 fois celle des bateaux. $\mathrm{La}$ voie d'eau est prévue pour la navigation d'automoteurs de $9,5 \times 80 \mathrm{~m}$ ou $11,4 \times 110 \mathrm{~m}$ et de convois. poussés à deux barges de $11,4 \times 185 \mathrm{~m}$ au total et un enfoncement de $2,5 \mathrm{~m}$. Le profil trapézoidal du canal offre une largeur en plan d'eau de $55 \mathrm{~m}$, une profondeur de $4 \mathrm{~m}$ au pied des talus à $3 / 1$ et de $4,25 \mathrm{~m}$ au milieu du canal. Il en résulte une section mouillée de $175,8 \mathrm{~m}^{2}$.

Quant à la construction du canal, les différents tronçons doivent dans toute la mesure du possible être réalisés en tranchée. Le dépôt des déblais excédentaires. coûte moins cher et comporte moins de risques que la réalisation de digues. $\mathrm{Si}$ les croisements du canal par des conduites véhiculant des produits liquides ne peuvent être déplacés de sections en remblais vers des sections en déblais ou dans les superstructures de ponts, il faut prendre de très amples mesures de sécurité. Une réalisation optimale de la construction doit englober également un système d'observation optimal.

\section{Besoins en eau pour le fonctionnement du canal}

Les écluses de haute chute sont des écluses avec des bassins d'épargne pouvant recevoir et restituer jusqu'à $60 \%$ du volume de remplissage du sas, contribuant 


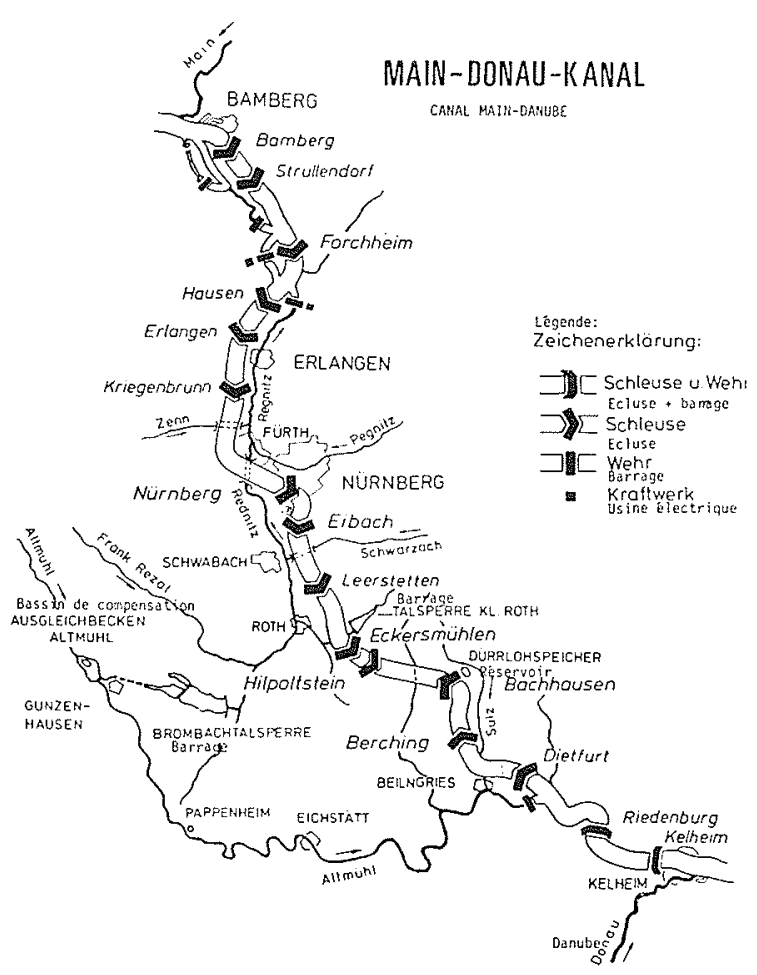

Figure 1. - Vue d'ensemble Bamberg-Kelheim Bild 1. - Ubersich tslageplan Bamberg-Kelheim

ainsi à une forte diminution du volume d'eau prélevé dans le bief supérieur.

Calculés sur la base de 96 remplissages par écluse et semaine, les besoins en eau de toutes les écluses entre Kelheim et Leerstetten s'élèveront à 4,0 millions de $\mathrm{m}^{3}$. Comme 1,6 millions de $\mathrm{m}^{3}$ retourneront au Danube et à l'Altmühl par l'intermédiaire des écluses sur le versant Sud, le Danube perdra 2,4 millions de $\mathrm{m}^{3}$ d'eau par semaine ou $3,9 \mathrm{~m}^{3} / \mathrm{s}$ en moyenne durant une semaine. A partir de l'écluse d'Eibach, la consommation d'eau sur le versant Nord, par suite des hauteurs de chute plus faibles, s'élèvera seulement à 1,9 millions de $\mathrm{m}^{3}$ par semaine. Si nous nous basons sur un total de 4500 éclusages par an, compte tenu des temps d'arrêt pour gel ou révision, nous arrivons à des pertes d'eau pour le Danube d'environ 112 millions de $\mathrm{m}^{3}$ par an. Ces pertes ne seront toutefois atteintes en totalité qu'après une période transitoire de plusieurs années après la mise en service de la voie d'eau, lorsque le volume du trafic évalué par le Groupe d'Etudes de la C.E.E. à 14 millions de $t$ /an sera atteint.

L'évaporation à la surface du plan d'eau viendra se substituer à celle qui se produisait dans les surfaces en végétation avant l'aménagement du canal. Suivant certaines évaluations, elle sera de moins d'un litre par seconde et par $\mathrm{km}$ et inférieure à $1 \%$, de l'étiage absolu de la Regnitz près de Hausen. Il ne s'agit en effet que d'une faible partie du bassin versant de la Regnitz et de l'Altmühl. Les pertes par infiltration seront très faibles, grâce à l'étanchéité soignée des parcours concernés, longs au total d'environ $70 \mathrm{~km}$. Tout apport éventuel aux eaux souterraines restera dans le bassin versant des deux cours d'eau. Les pertes d'eau survenant aux joints des portes des écluses et aux vannes d'aqueducs seront

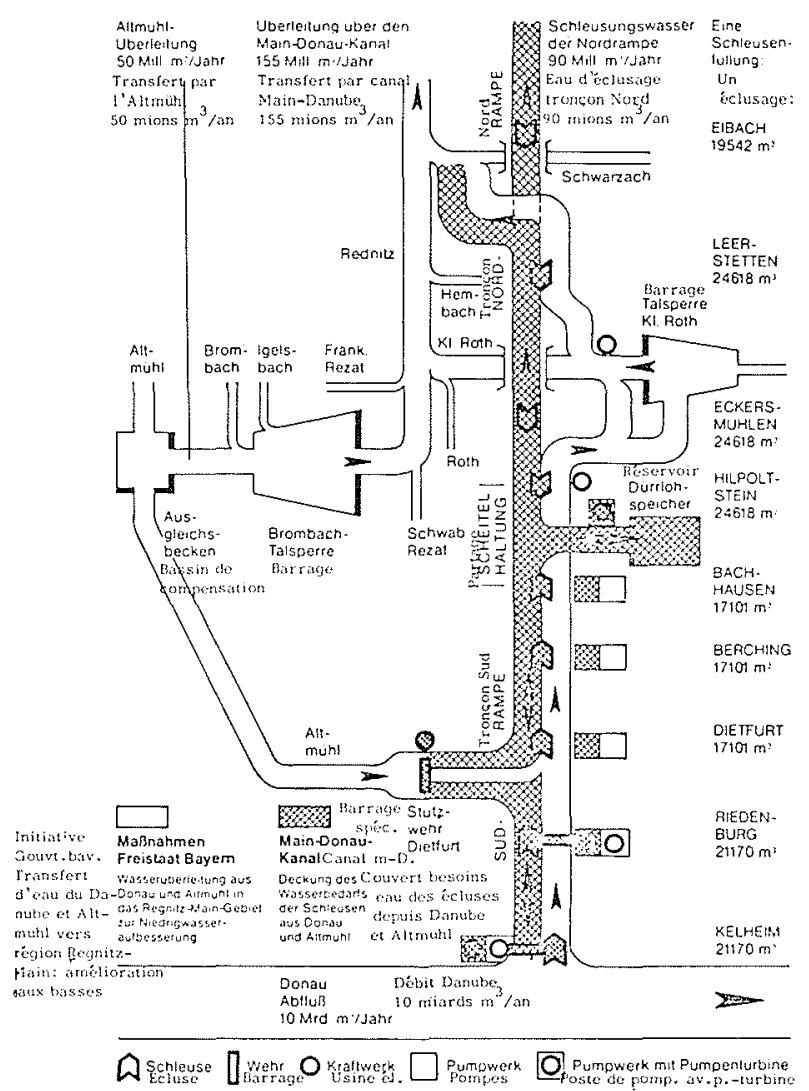

Figure 2. - Schéma du transfert d'eau vers la région RegnitzMain

Bild 2. - Schema der Uberleitung in das Regnitz-Main-Gebiet

compensées par l'action des stations de pompage des différents biefs.

\section{Couverture des besoins d'eau pour le fonctionnement du canal}

Les besoins en eau des écluses ne pourront pas être couverts par les apports d'eaux souterraines sur les tronçons sans revêtement étanche et par l'apport des cours d'eau. En 1921, l'année de fondation de la Société Rhin-Main-Danube, il était prévu de prélever l'eau nécessaire à l'alimentation du canal dans le Lech, affluent méridional du Danube, et de l'amener par une conduite d'environ $96 \mathrm{~km}$ de longueur par-delà du Danube, jusque dans le bief de partage. L'eau du Lech devait permettre non seulement le fonctionnement des écluses, mais également la production d'énergie hydro électrique. Le transfert d'eau devait porter sur un débit minimum de $31 \mathrm{~m}^{3} / \mathrm{s}$ dont une partie devait retourner au Danube. En 1922, la Commission du Danube, probablement parce qu'à l'occasion des travaux de construction du canal Main-Danube, le chenal de basses eaux du Danube devait être approfondi, a donné son accord à ce projet par une résolution.

Entre temps, on a construit dans le bassin versant du Danube une série de bassins de rétention qui servent à la protection contre les crues et en outre au soutien des étiages.

Dans les années 30 , on s'est rendu compte de ce qu'il était plus avantageux d'installer sur le Lech des usines 
hydro-électriques et de prélever, à l'aide du courant produit par ces usines - ou par des usines sur le Danube, mieux situées géographiquement - dans le Danube près de Kelheim, l'eau nécessaire au fonctionnement des écluses. Près de Kelheim, le volume d'eau annuel du Danube, en moyenne sur longue période, s'élève à 10 milliards de $\mathrm{m}^{3}$. Le débit d'étiage moyen y est de $140 \mathrm{~m}^{3} / \mathrm{s}$. L'eau du Danube est relevée jusqu'au bief de partage à l'aide de stations de pompage, par l'intermédiaire des deux biefs sur l'Altmühl et de 3 biefs en canal. On peut ainsi renoncer à une conduite spéciale pour l'alimentation du canal parce que l'eau est transférée, sur le versant Sud, de bief en bief.

\section{Postes de pompage sur le versant Sud}

Pour les postes de pompage sur le versant Sud, le débit d'équipement devait être tel qu'en règle générale les pompes ne travaillent que la nuit et le weekend et pas plus longtemps que 18 heures par jour, ce qui permettra de profiter de tarifs plus avantageux pour l'électricité. Pour cette raison, il y aura deux pompes pour assurer le fonctionnement des écluses, chaque pompe ayant un débit maximal de $7 \mathrm{~m}^{3} / \mathrm{s}$. Les stations de pompage pour les écluses sur l'Altmühl seront munies de pompes réversibles pour pouvoir fournir du courant électrique dans le secteur dès que le débit de 1'Altmüh1 sera suffisant.

\section{Réservoir de Dürrloh}

A l'extrémité amont du versant Sud, il est envisagé d'aménager, à côté de l'écluse de Bachhausen, le réservoir de Dürrloh. Etant donné les tarifs avantageux de nuit et surtout le samedi et le dimanche, cette installation aura le caractère d'un réservoir hebdomadaire. Le niveau d'eau du réservoir, qui sera plein le lundi matin, s'abaissera progressivement pour atteindre son niveau minimum vendredi après-midi; ensuite il $y$ aura remplissage au cours du weekend. Entre lundi et vendredi, le niveau d'eau variera en fonction des éclusages et $\mathrm{du}$ fonctionnement des pompes. S'il y a par jour ouvrable 16 éclusages, et le dimanche 8 , il faudra faire marcher les pompes même en dehors des heures à bas tarifs. Là aussi, des pompes réversibles permettront une production épisodique d'énergie électrique.

\section{Economie hydraulique sur un plan suprarégional}

Le bilan hydrologique de la Bavière est caractérisé par des contrastes hydrologiques et par l'évolution différente des besoins en eau dans le Nord et dans le Sud. Il faut donc pratiquer une économie hydraulique sur le plan suprarégional conformément à un mémoire élaboré en 1970 par l'Administration Centrale des Travaux Publics à Münich.

\section{Objectifs}

Les besoins en eau potable de la population doivent de préférence être couverts au moyen des eaux souter- raines et des eaux accumulées dansles barrages-réservoirs. L'industrie doit à l'avenir couvrir davantage ses besoins à partir des cours d'eau.

La qualité de l'eau des cours d'eau et des lacs doit être telle que ceux-ci ne servent pas seulement d'émissaires pour les eaux usées, mais soient en état d'assumer des rôles multiples.

Le long de certains cours d'eau, le risque des inondations, notamment au droit des agglomérations, doit être éliminé le plus largement possible.

Il faut créer les conditions nécessaires sur le plan de l'économie hydraulique pour le développement économique escompté dans la zone d'influence de la voie d'eau Main-Danube.

Dans l'intérêt de l'agriculture, il faut améliorer la structure agraire, y compris les systemes de drainage et d'irrigation.

\section{Besoins en eau de la région Main-Regnitz}

La Franconie centrale est relativement pauvre en eau et a un sous-sol présentant de faibles capacités de stockage d'eau. L'eau potable fait défaut. De nos jours déjà, les cours d'eau de cette région, en dépit d'investissements importants consentis par l'industrie et les communes pour des stations d'épuration d'eau, sont par moment très pollués. Les exigences relatives à la qualité de l'eau des rivières se sont considérablement renforcées durant les dernières décennies. De nouvelles quantités d'eaux usées ne peuvent plus être absorbées. Même une amélioration supplémentaire de l'épuration ne permettra plus d'obtenir une amélioration sensible de la situation. Donc, sans des apports d'eau supplémentaires, il n'est plus possible de créer ici de nouveaux emplois. Par contre, la région des Alpes et des Pré-Alpes est assez riche en précipitations atmosphériques et dotée d'un sous-sol en gravier se prêtant bien au stockage d'eaux souterraines, assurant ainsi des ressources en eau suffisantes.

\section{Couverture des besoins par transfert d'eau et système de transfert spécial}

Or, il est possible de transférer à l'avenir, vers la région Main-Regnitz, le long du cours inférieur de l'Altmühl et du versant Sud du canal Main-Danube et à l'aide de stations de pompage supplémentaires, de l'eau prélevée dans le Danube en lui faisant franchir la ligne de partage des eaux entre le Danube et le Rhin. Cela n'est pas seulement dans l'intérêt du développement économique de la Bavière septentrionale, mais constitue en même temps une condition essentielle pour le renouveau économique de la dite région, renouveau qui doit être obtenu grâce à l'importance que revêt la voie d'eau Main-Danube dans la fonction transport.

Pour ne pas troubler cependant le bilan hydrologique du Danube aux époques de faible débit, on envisage, en plus, l'installation d'un large système de transfert d'eau, dỉ système "Brombach". Ce système doit permettre de recueillir les crues de l'Altmühl dans le bassin de compensation près de Gunzenhausen et de diriger cette eau, par une conduite passant au moyen d'une galerie sous la ligne de partage Rhin - Danube, dans le barrage. réservoir de Brombach. L'eau y sera stockée la plupart 
du temps of sere, an monent on le dbit du Damube a

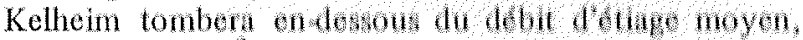
à savoir $140 \mathrm{~m}^{3} / \mathrm{s}$, transteres dun li Heon Roynit: Main par l'intermediaire de la Rezat ot do la Roduitz. Ainsi, les crues le long du cours moyen or inferiem do l'Altmühl seront atténuées à l'aide de co syatome lon même temps, il en résultera une augmentation du dóbit d'étiage. L'aménagement de l'Altmühl entre Gunzen" hausen et Pappenheim, depuis longtemps envisage, ne se justifiera économiquement qu'après la réalisation de ce système.

\section{Ouvrages de transfert d'eau}

Pour les besoins de l'économie hydraulique, 3 pompes réversibles d'une capacité unitaire de $7 \mathrm{~m}^{3} / \mathrm{s}$ seront installées dans les stations de pompage des biefs de Kelheim, Riedenburg, Dietfurt, Berching et Bachhausen.

Lorsque les pompes tournent à plein régime pour couvrir les besoins de la navigation et de l'économie hydraulique, la vitesse maximale d'écoulement dans le canal du versant Sud s'élève à $0,27 \mathrm{~m} / \mathrm{s}$. L'eau met au moins 6 jours pour arriver du Danube jusque dans le batrage-réservoir de la Roth. A un régime moindre des pompes, le temps d'écoulement peut être encore beaucoup plus long. Ces temps d'écoulement sont cependant sans importance pour les transferts d'eau par l'intermédiatro du canal parce que toutes les installations sont manouvrées de manière synchrone, ce qui permet de pretever a tout moment de l'eau dans le canal.

\section{Barrageteservoir de la Roth}

Le but principal du barrage-réservoir aménagé dans la vallée de la Kleinen Roth est de transformer en une alimentation uniforme sur 24 heures la partie des prélèvements opérés en 18 heures par le système de transfert par le canal. En outre, il est destiné à compenser de brèves interruptions dans la marche du système de transfert, lorsqu'un prélèvement d'eau dans le Danube n'est plus possible. Ayant une superficie de $2,12 \mathrm{~km}^{2}$, ce barrage-réservoir sera non seulement d'une grande importance pour l'économie hydraulique, mais également pour les sports nautiques et les loisirs.

Ce barrage recevra par l'intermédiaire du canal MainDanube en moyenne environ 105 millions de $\mathrm{m}^{3} / \mathrm{an}$. Il

Tableau 1 - Débits comparatifs Regnitz-Danube

Tabelle 1 - Abflussvergleich Regnitz-Donau

\begin{tabular}{|c|c|c|c|}
\hline $\begin{array}{c}\text { Vergleichswerte } \\
\text { Valeurs comparatives }\end{array}$ & $\begin{array}{l}\text { Einheit } \\
\text { Unité }\end{array}$ & $\begin{array}{l}\text { Regnitz Pegel Hüttendorf } \\
\text { Niveau d'eau Regnitz }\end{array}$ & $\begin{array}{l}\text { Donau Pege/ Kelheim } \\
\text { Niveau d'eau Danube }\end{array}$ \\
\hline $\begin{array}{l}\text { Niederschlags-Gebiet } \\
\text { Bassin versant } \\
\text { Niedrigster Abfluss (NNO) } \\
\text { Débit d'étiage mini } \\
\text { Mittlerer Niedrigabfluss (MNQ) } \\
\text { Débit d'étiage moyen } \\
\text { Mittlerer Abfluss } \\
\text { Débit moyen }\end{array}$ & $\begin{array}{l}\mathrm{km}^{2} \\
\mathrm{~m}^{3} / \mathrm{s} \\
\mathrm{m}^{3} / \mathrm{s} \\
\mathrm{m}^{3} / \mathrm{s}\end{array}$ & $\begin{array}{l}3870 \\
7.1^{*} \\
11.9^{*} \\
28,0\end{array}$ & $\begin{array}{r}22950 \\
85 \\
140 \\
321\end{array}$ \\
\hline
\end{tabular}

* $27.0 \mathrm{~m}^{3} / \mathrm{s}$ bei Uberleitung

* $27.0 \mathrm{~m}^{3} / \mathrm{s}$ en cas de transfert d'eau

Tableau 2 - Parcours de la liaison Main-Danube

Tabelle 2 - Abschnitte der Main-Donau-Verbindung

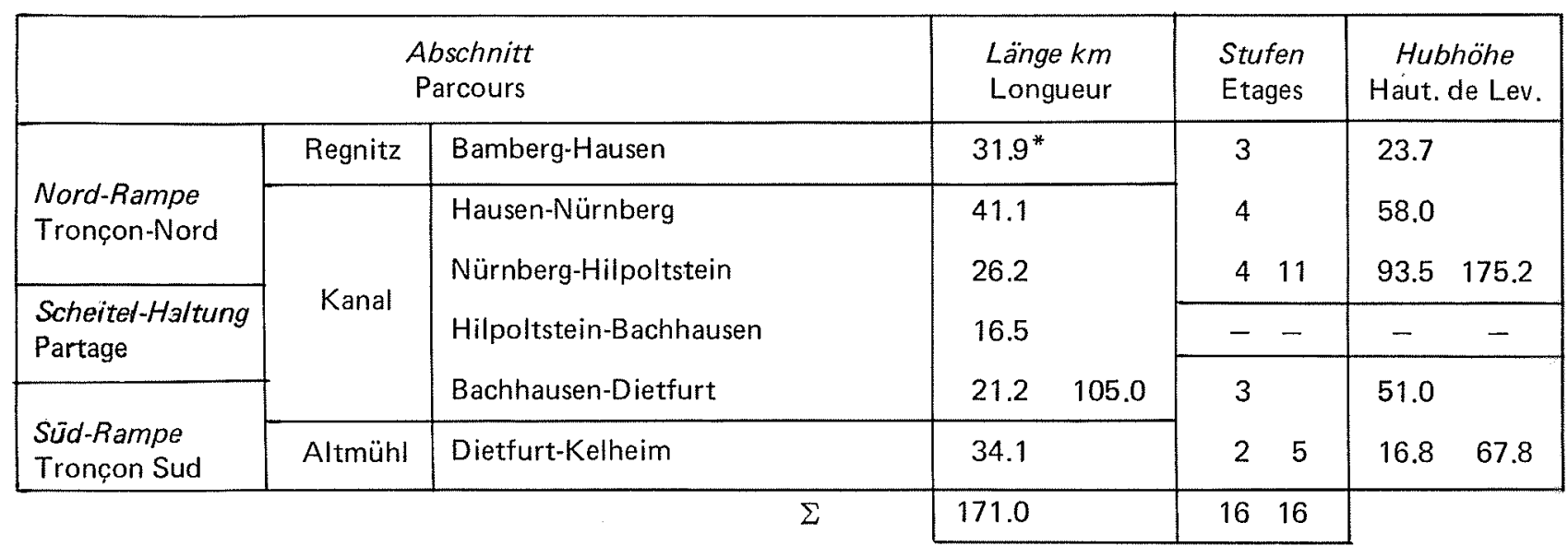

* davon $15.5 \mathrm{~km}$ Regnitz-Seitenkanal 
Tableau 3 - Ecluses $(12 \times 190 \mathrm{~m})$ de la liaison Main-Danube $(171 \mathrm{~km})$

Tabelle 3 - Schleusen $(12 \times 190 \mathrm{~m})$ der Main-Donau-Verbindung $(171 \mathrm{~km})$

\begin{tabular}{|c|c|c|c|c|c|c|c|c|c|c|}
\hline & $\begin{array}{c}\text { Ecluse } \\
\text { Schleuse }\end{array}$ & $\begin{array}{c}\text { Tête aval } \\
\text { Unterhaupt } \\
k m\end{array}$ & $\begin{array}{l}\text { Long. Bief } \\
\text { Haltungs- } \\
\text { länge } \mathrm{km}\end{array}$ & $\begin{array}{c}\text { Bief aval au- } \\
\text { dessus ni- } \\
\text { veau mer } \\
\text { Unter } \\
\text { Wasser } \\
m \text { ü.NN }\end{array}$ & $\begin{array}{c}\text { Hauteur de } \\
\text { levage } \\
\text { Hubhöhe m }\end{array}$ & $\begin{array}{c}\text { Bief amont } \\
\text { au-dessus ni- } \\
\text { veau mer } \\
\text { Obserwasser } \\
m \text { ï.NN }\end{array}$ & $\begin{array}{c}\text { Bassins } \\
\text { d'épargne } \\
\text { Sparbecken } \\
\text { Anzahi }\end{array}$ & $\begin{array}{l}\text { Reste d'eau } \\
\text { de remplis- } \\
\text { sage à } \\
\text { fournir } \\
\text { Restfüll- } \\
\text { Wasser- } \\
\text { menge } m^{3}\end{array}$ & $\begin{array}{r}\text { Bes } \\
\text { pa } \\
W a \\
J \\
M i o \\
m^{3}\end{array}$ & $\begin{array}{l}\text { ns d'eau } \\
\text { emaine } \\
\text { abedarf } \\
\text { Voche } \\
m^{3} / s \\
\text { moyenne } \\
\text { I.M. }\end{array}$ \\
\hline 1 & Bamberg & 7,3 & 5,8 & 230.8 & 11,0 & 241,8 & 3 & 11294 & 1,1 & 1,8 \\
\hline 2 & Strullendorf & 13,1 & 12,8 & 241,8 & 7,4 & 249,2 & 1 & 11185 & 1,1 & 1.8 \\
\hline 3 & Forchheim & 25,8 & 7,0 & 249,2 & 5,3 & 254,5 & - & 12720 & 1,2 & 2.0 \\
\hline 4 & Hausen & 32,9 & 8,2 & 254,5 & 12,0 & 266,5 & 2 & 15031 & 1,9 & 3,1 \\
\hline 5 & Erlangen & 41,1 & 7,6 & 266,5 & 18,3 & 284,8 & 3 & 18433 & 1,9 & 3,1 \\
\hline 6 & Kriegenbrunn & 48,7 & 20,6 & 284,8 & 18,3 & 303,1 & 3 & 18433 & 1,9 & 3,1 \\
\hline 7 & Nürnberg & 69,3 & 3,7 & 303,1 & 9,4 & 312,5 & 1 & 15598 & 1,9 & 3,1 \\
\hline 8 & Eibach & 73,0 & 11,4 & 312,5 & 19,5 & 331,99 & 3 & 19605 & 1,9 & 3,1 \\
\hline 9 & Leerstetten & 84,4 & 10,7 & 331,99 & 24,67 & 356,66 & 3 & 24618 & 2,4 & 3,9 \\
\hline 10 & Eckersmühlen & 95,1 & 4,0 & 356,66 & 24,67 & 381,33 & 3 & 24618 & 2,4 & 3,9 \\
\hline 11 & Hilpoltstein & 99,1 & 4 & 381,33 & 24,67 & 406,0 & 3 & 24618 & 2,4 & 3,9 \\
\hline 12 & Bachhausen & 115,6 & & 389,0 & 17,0 & 406,0 & 3 & 17101 & 1,6 & 2,7 \\
\hline 13 & Berching & 122,6 & 7,0 & 372,0 & 17,0 & 389,0 & 3 & 17101 & 1,6 & 2,7 \\
\hline 14 & Dietfurt & 135,4 & 12,8 & 355,0 & 17,0 & 372,0 & 3 & 17101 & 1,6 & 2,7 \\
\hline 15 & Riedenburg & 151,0 & 15,6 & 346,6 & 8,4 & 355,0 & - & 21170 & 2,0 & 3,4 \\
\hline 16 & Kelheim & 166,1 & 15,1 & 338,2 & 8,4 & 346,6 & - & 21170 & 2,0 & 3,4 \\
\hline
\end{tabular}

A/tmüh/mündungsstrecke

Embouchure Altmühl

4,9

Tableau 4 - Barrages, bassins et réservoirs pour la navigation et l'économie hydraulique le long de la liaison Main-Danube Tabelle 4-Spoicherbecken für schiffahrt und wasserwirtschaft im bereich der Main-Donau verbindung

\begin{tabular}{|c|c|c|c|c|c|c|}
\hline $\begin{array}{l}\text { Speicherbecken } \\
\text { Barrages, bassins, réservoirs }\end{array}$ & $\begin{array}{c}\text { Stauziel } \\
m \text { ü.NN } \\
\text { Retenue } \\
\text { normale } \\
\text { au-dessus } \\
\text { niveau mer }\end{array}$ & $\begin{array}{c}\text { Absenkziel } \\
m \ddot{u} . N N \\
\text { Retenue } \\
\text { mini } \\
\text { au-dessus } \\
\text { niveau mer }\end{array}$ & $\begin{array}{l}\text { Wasser- } \\
\text { fläche } \\
k m^{2} \\
\text { Superficie } \\
\text { d'eau }\end{array}$ & $\begin{array}{l}\text { Uberlänge } \\
k m \\
\text { Longueur } \\
\text { Bords }\end{array}$ & $\begin{array}{l}\text { Speicher- } \\
\text { kapazität } \\
\text { Mio } m^{3} \\
\text { Capacité }\end{array}$ & $\begin{array}{l}\text { Funktion des } \\
\text { Speicherbeckens } \\
\text { Fonction }\end{array}$ \\
\hline $\begin{array}{l}\text { Altmühl-Ausgloichbecken } \\
\text { bei Gunzenhausen } \\
\text { Bassin de compens. près Gunzenhausen } \\
\text { Brombach-Talsperre } \\
\text { Barrage du Brombach }\end{array}$ & 411,0 & 403,5 & 11,74 & 30 & 67,0 & $\begin{array}{l}\text { Hochwasserableitung und } \\
\text { niedrigwassererhöhung } \\
\text { Evacuation crues et } \\
\text { amélioration eaux basses } \\
\text { Jahrésspeicher } \\
\text { Réservoir annuel }\end{array}$ \\
\hline $\begin{array}{l}\text { Roth-Talsperre } \\
\text { Barrage de la Roth }\end{array}$ & 374,2 & 367,2 & 2,12 & 10 & 7,1 & $\begin{array}{l}\text { Wochenspeicher } \\
\text { Réservoir hebdomadaire }\end{array}$ \\
\hline $\begin{array}{l}\text { Kanal-Scheitelhaltung } \\
\text { Bief de partage canal }\end{array}$ & 406,3 & 406,0 & 0,91 & 33 & 0,3 & $\begin{array}{l}\text { Tagesspeicher } \\
\text { Réservoir journalier }\end{array}$ \\
\hline $\begin{array}{l}\text { Kanalspeicher Dürrloh } \\
\text { Réservoir }\end{array}$ & 415,5 & 407,7 & 0,24 & 2 & 1,7 & $\begin{array}{l}\text { Wochenspeicher } \\
\text { Réservoir hebdomadaire }\end{array}$ \\
\hline $\begin{array}{l}\text { Donaustauraum Straubing } \\
\text { Volume de retenue Danube }\end{array}$ & 320,3 & 319,7 & 5,70 & 50 & 3,0 & $\begin{array}{l}\text { Abflussausgleich je } \\
\text { Woche } \\
\text { Compens, débit mensuel }\end{array}$ \\
\hline
\end{tabular}


est prévu de porter plus tard cette quantité au double à peu près.

\section{Bassin de compensation de l'Altmühl}

Le bassin de compensation projeté dans une cuvette de $4 \mathrm{~km}$ de large dans la vallée de l'Altmühl entre Ornbau et Gunzenhausen, constitue un élément important đu système "Brombach". Il servira à opérer en très peu de temps la rétention d'environ 50 millions de $\mathrm{m}^{3}$ d'eau par an et à fournir, suivant la capacité de la conduite de transfert, un maximum de $70 \mathrm{~m}^{3} / \mathrm{s}$ d'eau. La superficie de $5 \mathrm{~km}^{2}$ servira aussi aux sports nautiques et à la baignade. D'autre part cette région a aujourd'hui déjà une grande importance comme zone de nichage et d'étape pour les oiseaux aquatiques, raison pour laquelle de larges parties du bord de ce bassin de même qu'une 1̂le seront conçues de manière à permettre la formation d'une bande de roseaux naturelle.

\section{Barrage -éservoir de Brombach}

Avec une superficie de $11,74 \mathrm{~km}^{2}$ et une hauteur maxi de retenue de $35,5 \mathrm{~m}$ au-dessus du fond de la vallée, ce barrage a des dimensions considérables et ne manquera pas d'influencer sensiblement le paysage et la structure de cette région pauvre en eau. Il dépassera la superficie du lac de Tegernsee couvrant $8,9 \mathrm{~km}^{2}$. La retenue normale de $411,0 \mathrm{~m}$ au-dessus du niveau de la mer permettra de stocker un volume total de 67 millions de $\mathrm{m}^{3}$ d'une part dans le volume utile du barrage et d'autre part dans la partie supérieure servant à la protection contre les crues. Un déversoir permettra l'évacuation de $25 \mathrm{~m}^{3} / \mathrm{s}$, au besoin même de $50 \mathrm{~m}^{3} / \mathrm{s}$. Après le remplissage de ce barrage, il s'y produira bientôt une eutrophisation, mais qui, probablement dans les trois ans qui suivent, redisparaîtra lorsque les matières biodégradables seront extraites du fond de la vallée et détruites. Par la mise en place de collecteurs et d'une installation d'épuration en aval du barrage, il sera possible d'em- pêcher le rejet d'eaux usées. Les bassins secondaires sur le Brombach et l'Igelsbach, qui se prêteront bien aux loisirs, ne subiront pas d'importantes variations du niveau d'eau.

\section{Résultats et perspectives}

Le canal Main-Danube, qui reliera probablement à la fin des années quatre-vingt le Danube au Rhin, ne constituera pas seulement la voie d'eau la plus importante d'Europe centrale. Il permettra également une large opération de reéquilibrage hydrologique grâce d'une part au système de transfert d'eau par le canal et d'autre part au système du Brombach qui sera terminé quelques années plus tôt. Ces deux buts se complètent l'un l'autre dans l'intérêt de l'économie globale. Les frais de construction du tronçon Nuremberg-Kelheim sont évalués à 1800 millions de DM, ceux des deux systèmes de transfert, à 640 millions de DM. La commande de toutes les installations, qui sera assurée par une centrale projetée à Nuremberg, sera effectuée en fonction de la navigation et des besoins hydrauliques. La qualité de l'eau dans les deux systèmes de transfert jouera également un rôle certain.

\section{Reférences}

[1]FUCHS H. - Wasserwirtschaftliche Probleme des MainDonau-Kanals. Selbstverlag der Rhein-Main-Donau AG München 1966.

[2] Oberste Baubehörde im Bayerischen Staatsministerium des Innern : Uberleitung von Altmühl -und Donauwasser in das Regnitz-Main-Gebiet. Selbstverlag München 1970.

[3] KUHN R. und andere. - Alimentation en eau des voies d'eau, notamment des canaux à bief de partage, compte tenu des différentes utilisations de l'eau. Deutsche Berichte zum XXIV. Internationalen Schiffahrtskongress Leningrad 1977.

[4] Wasserverband Westdeutscher Kanäle : Schiffahrtskanäle als Fernwasserleitungen. Selbstverlag Essen 1980. 